\title{
A local view on the role of friction and shape
}

\author{
Matthias Schröter ${ }^{1, \star}$ \\ ${ }^{1}$ Institute for Multiscale Simulation, Friedrich-Alexander-University (FAU), 91052 Erlangen, Germany
}

\begin{abstract}
Leibniz said "Naturam cognosci per analogiam": nature is understood by making analogies. This statement describes a seminal epistemological principle. But one has to be aware of its limitations: quantum mechanics for example at some point had to push Bohr's model of the atom aside to make progress. This article claims that the physics of granular packings has to move beyond the analogy of frictionless spheres, towards local models of contact formation.
\end{abstract}

On earth solid assemblies of granular particles are by far the most frequent phase of granular matter; we encounter granular packings everywhere from our kitchen cabinet to civil engineering textbooks. In order to make their handling, transport, and storage more efficient, we strive for a theory that predicts their mechanical properties, such as shear and bulk modulus or yield stress, starting from a few state variables only. Efforts to develop such a theory often start by modeling granular packings as an assembly of frictionless spheres. This is a rather unsuitable starting point, for a number of reasons:

1. All granular particles are frictional.

2. Frictional particles have lower isostatic numbers than frictionless particles.

3. Granular physics happens at volume fractions inaccessible to frictionless particles.

4. The volume fraction of soft particles can be changed by compression. The volume fraction of frictional particles is changed by changing their geometry.

5. Friction is one reason for history dependence in granular systems.

6. Real world granular media are rarely spherical. Shape adds complexity, e.g. to history dependence.

These six theses are also the outline for the following sections. They are intended to provoke discussions with a sizeable subgroup of the theoretically or numerically working scientists. Many experimentalists, applied scientist, and engineers might find them, at least in part, well-known. For simplicity, we will discuss in the following only monodisperse spheres; except for section 6 .

\section{All granular particles are frictional.}

Contrary to other particulate systems, such as foams or emulsions, the constituents of granular media are solid particles. This implies that their surface is geometrically

\footnotetext{
^e-mail: matthias.schroeter@fau.de
}
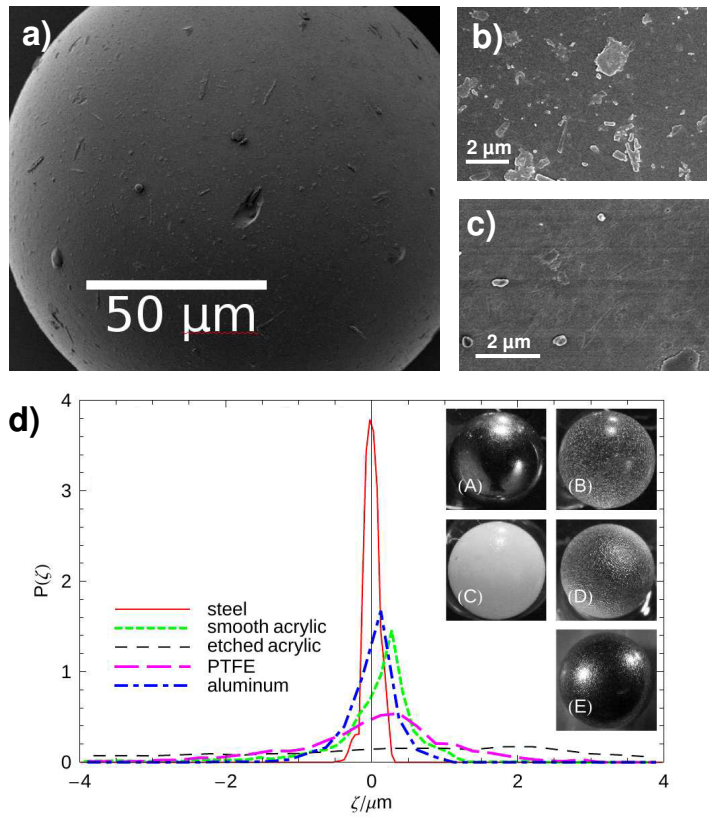

Figure 1. Granular particles are rough particles. a) Scanning Electron microscope (SEM) image of a factory-new soda-lime glass bead. Image by courtesy of Karina Sand. b) SEM image of the asperities on the surface of a new soda-lime glass bead. c) After 30900 flow pulses in a water fluidized bed, abrasion has removed many of the surface asperities, resulting in a measurable difference in packing properties. Images b) and c) are taken from [1]. d) Histograms of surface roughness $\xi$ of spherical particles measured with a profilometer. The inset shows images of the corresponding particles: (A) steel, (B) smooth acrylic, (C) PTFE, (D) solvent-etched acrylic, (E) aluminum. Reproduced from [2].

rough, cf. figure 1. If two particles get into contact, their surface asperities will interlock, allowing for the existence of tangential forces at the contact [3]. In the context of granular packings, friction is sufficiently well described by 

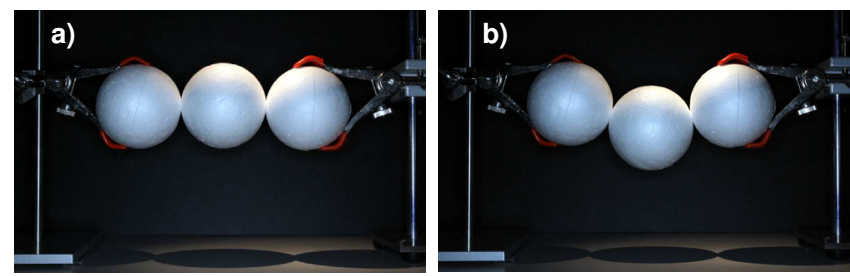

Figure 2. Friction increases the number of mechanically stable configurations. In both images the center Styrofoam sphere is only hold in place by the tangential forces at the contacts. Neither arrangement would be possible with frictionless spheres. In fact, under gravity the only mechanically stable configuration of three frictionless spheres is the perfect vertical alignment.

the Amontons-Coulomb law:

$$
F_{t} \leq \mu F_{n}
$$

$F_{n}$ and $F_{t}$ are the normal and tangential components of the contact force, and $\mu$ is the static coefficient of friction.

All granular media consist of frictional particles. Even hydrogel spheres, which consist of up to $99.5 \%$ water, have a friction coefficient of $\approx 0.01[4,5]$. Moreover, while it is possible to relax all tangential forces in a packing by vibrating it at small amplitudes and high frequencies [6], this will also compactify the packing to values falling into the range of frictionless packings, thereby bypassing the interesting granular physics as discussed in section 3 .

In loose granular systems, such as e.g. granular gases, the dynamics is more controlled by collisions than contacts. Friction changes the way particles exchange momentum during collisions, but this seems to be often only a higher order perturbation. Granular packings on the other hand consist of enduring contacts, here the existence of friction changes the physical picture completely:

First, the presence of tangential forces provides additional ways to satisfy the force and torque balance, which will be discussed more quantitatively in section 2 . This leads to a massive increase of the number of mechanically stable states; figure 2 gives a simple example. Most granular packings studied in nature or experiment are looser than the loosest packing that can be created without friction. The consequences of this will be discussed in sections 3 and 4.

Secondly, equation 1 is an inequality. For a given normal force at a contact it allows for a whole range in tangential force, as shown in figure 3 . The actual tangential force will be a consequence of how the contact was formed. This property is one of the reasons for the so called historydependence of granular matter, which will be discussed further in section 5 .

\section{Frictional particles have lower isostatic numbers than frictionless particles.}

For any granular packing to be solid, the average number of contacts a particle forms with its neighbors $Z$ has to be at least so large that all degrees of freedom (DOF) of the

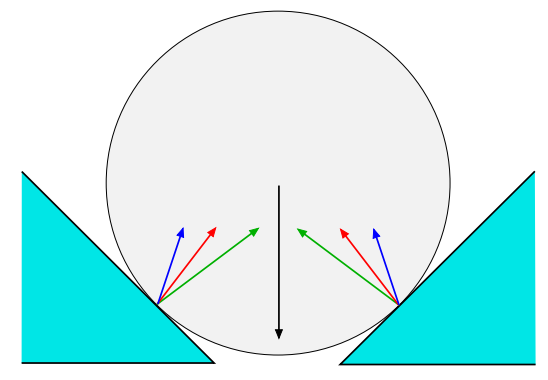

Figure 3. The contact forces of a sphere in a wedge depend on the preparation history. Each of the blue, red, or green pairs of contact forces can balance the weight of the sphere (black arrow). Which one is realized depends how the sphere was placed.

particle can be fixed. This minimal value, the so called isostatic contact number $Z_{i s o}$, does depend on the dimension, shape, and most importantly friction of the particles considered.

In the absence of friction, the rotational DOF of a perfect sphere are not relevant and only the three translational DOF have to be blocked by the contacts. At each contact there exists one normal force, which is however "shared" between the two particles which means that each contact blocks on average only 0.5 DOF per particle. A packing of frictionless spheres needs therefore to have at least $Z_{i s o}^{0}=6$ contacts to be mechanically stable.

If we assume $\mu=\infty$, then each contact has 3 independent force components (one normal and two tangential), which fix 1.5 constraints per particle. On the other hand, we now also have to consider the rotational DOF which results in 6 DOF per particle. The isostatic number in the presence of infinite friction $Z_{i s o}^{\mu}$ is therefore 4.

The inequality $Z_{i s o}^{0}>Z_{i s o}^{\mu}$ holds for all particle shapes and in 2 and 3 dimensions [7]. One consequence is the massive increase in the number of mechanically stable packings of frictional particles, as discussed in the next section. Another consequence is that granular packings are typically hyperstatic i.e. their actual contact number is larger than the minimum number needed for stability: $Z>Z_{i s o}^{\mu}$. From this follows that for a given spatial configuration of particles, there exists a multitude of possible force networks that will all satisfy the boundary conditions of the system [8]. This property is intimately connected to the history dependent behavior discussed in section 5 .

There is a possible caveat regarding hyperstaticity. In real granular media $\mu$ is finite and the contacts might have tangential forces which are exactly at the Coulomb threshold, so called fully mobilized contacts. As this type of contact will block only $1 \mathrm{DOF}$, the constraint counting argument has to be modified and the effective $Z_{i s o}^{\mu}$ becomes larger. However, a number of numerical studies [9-12] have shown that the number of fully mobilized contacts is not sufficient to regain isostaticity in any other situation than when preparing a pressure free packing very slowly, cf figure 4. Which is also the recipe to approach the limit of Random Loose Packing, the loosest packing possible (discussed in section 3.2). 


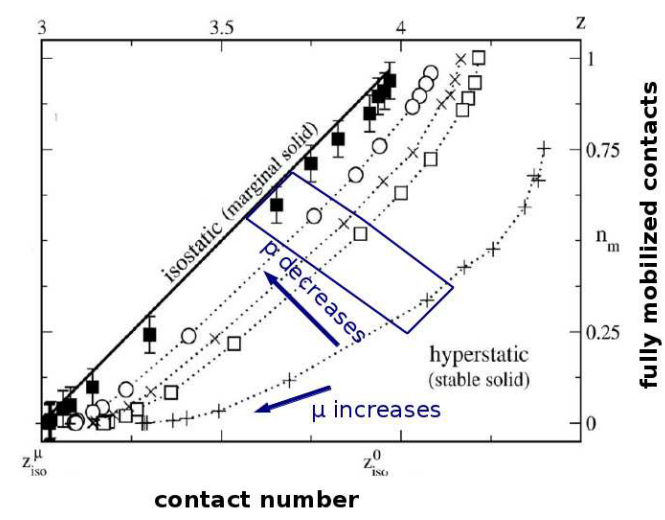

Figure 4. Most granular packing are also hyperstatic when fully mobilized contacts are taken into account. In disc packings both $Z$ (x-axis) and the number of fully mobilized contacts (y-axis) changes with pressure and $\mu$. However, only in the limit of vanishing pressure the system will approach isostaticity (solid line). From [9].

\section{Granular physics happens at volume fractions inaccessible to frictionless particles.}

The consequences of the lower isostatic contact number of frictional particles are best discussed using the the concept of the configurational entropy $S_{\text {conf }}$ of the packings. $S_{\text {conf }}$ was first introduced by Sam Edwards [13, 14], it is proportional to the logarithm of the the number of mechanically stable packing configurations that fit in a given volume and support given boundary conditions. As we are interested in the thermodynamic limit, we will discuss here $S_{\text {conf }}$ as a function of the global volume fraction $\phi_{g}$. More specifically, we are interested in a comparison of the upper and lower bounds of $\phi_{g}$ between which $S_{\text {conf }}$ becomes nonzero for both frictional and frictionless systems.

The main results of this discussion are summarized in figure 5. But a word of caution is necessary: The $\phi_{g}$ values of the upper and lower bounds are well supported by the numerical and experimental work discussed below. But the functional form of $S_{\text {conf }}$ connecting these boundaries is speculative and only supported by the heuristic arguments given below.

Moreover, we are only interested in packings that exist in a thermodynamic sense. This excludes both packings crystallizing in FCC and HCP configurations at volume fractions above $\phi_{g} \approx 0.65[15-21]^{1}$ and the "tunneled crystal" packings at $\phi_{g}=0.49$ [22]. Neither of these two configurations are extensive, i.e. their number does not grow exponentially with the number of particles in the system. Which means that in the thermodynamic limit their entropy is zero.

\footnotetext{
${ }^{1}$ Readers familiar with equilibrated hard sphere systems might expect crystallization to occur in the range $\phi_{g} \approx 0.494-0.61$. However, these systems are driven by the entropy increase due to newly gained vibrational DOF. These DOF do not exist in athermal granular packings where all particles are permanently in contact.
}

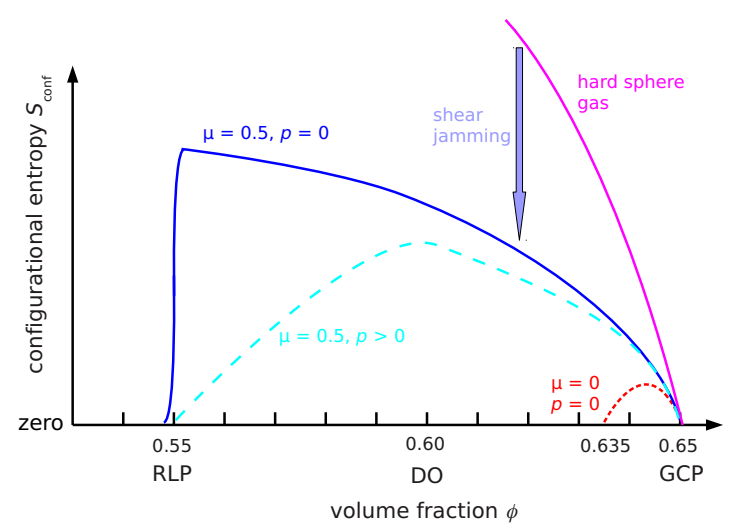

Figure 5. A schematic how the configurational entropy $S_{\text {conf }}$ of a sphere packing depends on the friction coefficient $\mu$ and the confining pressure $p$. The solid magenta line indicates the configurational entropy of an amorphous hard sphere gas i.e. a "packing" with non overlapping particles but no requirements on the mechanical stability or number of contacts. The solid blue and dashed cyan line represent packings with an approximate real world value of $\mu$ and the dotted red line corresponds to frictionless particles. While the points with $S_{\text {conf }}=0$ are well supported by experiments and simulations, the actual shape of the different curves is speculative.

An important upper bound on $S_{\text {conf }}$ is the configurational entropy $S_{H S}$ of an amorphous hard sphere gas where the only remaining condition for a valid configuration is that particles do not overlap. Mechanical stability and consequentially $Z$ do not matter. In figure 5 this boundary is indicated by a solid magenta line. The pressure of a hard sphere gas diverges at the so called Glass Close Packing (GCP) point with $\phi_{G C P} \approx 0.65[20,21,23]$. From which follows that the system runs out of nonoverlapping configurations and $S_{H S}$ goes to zero.

\subsection{Frictionless sphere packings: $0.635<\phi_{g}<0.65$}

As $S_{H S}$ is an upper limit for any sphere packing, $S_{\text {conf }}$ of a frictionless packing also needs to go to zero at $\phi_{G C P} \approx$ 0.65 . For any smaller value of $\phi_{g}, S_{\text {conf }}$ has to be smaller than $S_{H S}$ because we now additionally require an isostatic number of contacts. In fact, the set of mechanically stable configurations should be of measure zero compared to hard sphere gas: there are infinite more possibilities of two spheres to be not in contact compared to the one configuration where they are. Luckily, we know that $S_{\text {conf }}$ is still extensive. This was shown for soft frictionless disk and sphere systems of different sizes by dividing the total accessible phase space volume by that of an average basin of attraction [24, 25].

The total range of $S_{\text {conf }} \neq 0$ is shown as a red dotted line in figure 5. There is still some debate [7, 21, 26, 27] if the onset of mechanical stability happens for frictionless spheres at the so called Jamming point of $\phi_{J} \approx 0.64$ or slightly below at $\phi_{g} \approx 0.635$. However, it is known that the actual volume fraction of a packing of uncompressed 
frictionless spheres will depend on the preparation history. For an extended discussion and further references see [28].

\subsection{Frictional sphere packings: $0.55<\phi_{g}<0.65$}

All mechanically stable configuration of frictionless particles will stay valid if we allow for additional tangential forces. Therefore $S_{\text {conf }}$ of frictional packings will always be larger than its frictionless counterpart. Because $S_{H S}$ is also an upper bound to frictional systems, GCP will still be the upper limit for uncompressed packings.

The lower boundary, commonly referred to as Random Loose Packing (RLP), is however considerably lower than in frictionless systems: As mentioned in the last section, the inequality $Z_{i s o}^{0}>Z_{i s o}^{\mu}$ holds for all particle shapes and in 2 and 3 dimensions. Moreover, as $Z$ can be generically expected to decrease monotonically with decreasing $\phi_{g}$ (i.e. larger average separation between particles), the onset of mechanical stability will happen at a lower volume fraction for frictional particles. The actual value of $\phi_{\mathrm{RLP}}$ does depend on pressure [29, 30] and the friction coefficient $[2,30]$ : the higher $\mu$ the smaller is $\phi_{\mathrm{RLP}}$. For the experimentally common values of $\mu \approx 0.5$ and vanishing pressure, $\phi_{\mathrm{RLP}}$ approaches 0.55 [2, 29-33].

In figure 5 the solid blue and the dashed cyan line represent $S_{\text {conf }}$ of sphere packing with a finite value of $\mu$ and either zero or finite confining pressure $p$. Besides the points with $S_{\text {conf }}=0$, the shape of the curves is speculative as there are few analytical or experimental results on how $S_{\text {conf }}$ depends on $\phi_{g}$. What we do understand is that for any given value of $\phi_{g}, S_{\text {conf }}$ will grow monotonically with $\mu$ because allowing larger tangential forces will never destabilize any existing packing, but allow for new, additional configurations [34]. Moreover, we can use the Widom insertion method in combination with experimental or numerical packings to obtain an upper bound on $S_{\text {conf }}$ [35]. However, a lower bound would be more helpful. Finally, under certain additional assumptions, $S_{\text {conf }}$ can be computed from the volume fluctuations of a repeatedly driven granular packing [36-38]. However, the results obtained this way do not agree with each other.

Another way of assessing the shape of $S_{\text {conf }}\left(\phi_{g}\right)$ is to consider experimental preparation protocols and to use the additional assumption that the state the system will end up in is the most likely one: the one with the highest value of $S_{\text {conf }}$ under the given circumstances. For example, packing prepared by slow sedimentation in an almost density matched fluid (i.e. in the limit $p \rightarrow 0$ ) will always end up at $\phi_{\text {RLP }}[2,29,30]$. Which indicates that $S_{\text {conf }}$ has a maximum at RLP for $p=0$, cf. the blue line in figure 5 .

Without density matching (i.e. at a finite static pressure) and with an increased sedimentation speed (meaning that the settling particles will transfer more momentum on the already existing packing), the most likely packing fraction moves up to $\phi \approx 0.6$ [1]. The cyan dashed line in figure 5 represents the idea that this becomes the new maximum in $S_{\text {conf }}$. Which can be rationalized by assuming that configurations with less excess contacts compared to an isostatic packing are more likely to not posses a force network capable of supporting the increased stress at the boundaries. $\phi \approx 0.6$ is incidentally also the value for the onset of dilatancy, which is discussed in the next subsection.

Finally, getting the system to compactify to values of $\phi_{g}$ above 0.6 requires repeated driving under confining gravitational pressure, either by flow pulses [1] or mechanical taps $[39,40]$. This indicates that these states become more and more unlikely which agrees with the idea that $S_{\text {conf }}$ goes to zero for $\phi_{g}$ approaching GCP.

But the main point of this section is untouched by this discussion of the shape of $S_{\text {conf }}$ : The range of volume fractions of mechanically stable packings is seven times larger for frictional particles than for frictionless particles.

\subsection{Dilatancy in frictional packings}

Most of the interesting physics of granular packings happens at intermediate values of $\phi_{g}$ (i.e. between RLP and GCP); these volume fractions are inaccessible to frictionless packings. A good example is dilatancy: If a dense granular packing is sheared (at a finite hydrostatic pressure), it will expand [41]. However, dilatancy does not occur in sufficiently loose samples; those will instead compactify.

Now if dense packings expand during shear and loose packings collapse, there will be an intermediate density, usually termed Dilatancy Onset (DO) or critical state, where the volume fraction $\phi_{D O}$ stays constant during shear. Due to effects like shear banding the exact determination of $\phi_{D O}$ is not straightforward, but most experiments point to $\phi_{D O} \approx 0.6$ for frictional spheres at comparatively small confining pressures [42-47]

Dilatancy is closely related to a phenomenon labeled shear-jamming [48-50]. For $\phi_{g}>\phi_{D O}$ one can start from a hard sphere gas configuration (experiments are done in horizontal two-dimensional system and therefore effectively in the absence of gravity), shear it at a constant volume and arrive at a mechanically stable configuration. This protocol is indicated as a blue arrow in figure 5. Below $\phi_{D O}$ this is not possible.

One way of interpreting $\phi_{D O}$ is to see it as a "natural attractor" for all sheared systems starting at other volume fractions. This interpretation agrees well with the idea of $S_{\text {conf }}$ being maximal at $\phi_{D O} \approx 0.6$.

\section{The volume fraction of frictional particles is controlled by their geometry.}

The exact value of $\phi_{g}$ of a frictionless packing at zero pressure does depend on the preparation history [20, 21, 28, 51]. However, the scaling laws of these packings are normally studied by preparing pressure free packings with a given protocol and then increasing $\phi_{g}$ by compressing this packing $[7,26]$.

With respect to the contact number $Z$ this amounts to a study of the pair correlation function, or more precisely the slope of the right shoulder of the first peak which describes 
the close-by particles which will form contacts when compressed. This slope leads to an equation for $Z$ :

$$
Z\left(\phi_{g}\right)=Z_{i s o}^{0}+c\left(\phi_{g}-\phi_{i s o}\right)^{0.5}
$$

Here $\phi_{i s o}$ is the volume fraction of the uncompressed, isostatic packing and the constant $c$ depends on the dimension and polydispersity of the system. For compressed frictionless packings such as emulsions and foams [52], equation 2 is indeed a good description.

However, real world granular particles are normally not very squishy; they change their volume fraction by isobarically changing their packing geometry not by compression. We all intuitively know this from kitchen physics: if we want to fill more grains into a storage container we do not compress them with a piston, but we tap the container a couple of times on the counter top to change its packing structure.

For a more quantitative example lets compare two glass spheres (Young's modulus $=70 \mathrm{GPa}$, diameter $=250$ $\mu \mathrm{m})$ which are either uncompressed at the upper surface or compressed below a $1 \mathrm{~m}$ high column of other glass spheres. Using Hertz law we can derive that this increase in pressure will deform the sphere by approximately $10 \mathrm{~nm}$ at each contact. This deformation is an order of magnitude smaller than the vertical surface roughness of typical glass spheres[53]. Assuming that the sphere is compressed symmetrically, this corresponds to a change in volume fraction of $7 \times 10^{-5}$ compared to the uncompressed sphere. This illustrates that the large range of $0.55<\phi_{g}<0.65$ available to frictional sphere packings can not be explored by compression.

Please note that granular experiments can be performed in a way to test frictionless models. E.g. the compression of frictional but sufficiently soft photoelastic discs (with a Young's modulus of 4MPa [54]) can be used to verify equation 2 [54] or study glassy behavior [55]; provided that the tangential forces are relaxed by additional tapping or vibration. These experiments do however not prove that frictionless models describe generic frictional particles.

\subsection{Friction with your neighbors? Think locally!}

Because $Z$ and $\phi_{g}$ are in frictional packings not simultaneously controlled by the globally defined parameter pressure, the idea expressed in equation 2 of a function $Z\left(\phi_{g}\right)$ runs into an epistemological problem. Contacts are formed at the scale of individual particles and their neighbors. At this scale the global $\phi_{g}$ is not only undefined; due to local volume correlations $[56,57]$ it would even be impossible for a particle scale demon to compute $\phi_{g}$ by averaging over the volume of the neighboring particles.

What is needed for the theoretical description of frictional particles is an ansatz which explains $Z$ using only locally defined (i.e. on a particle level) parameters [5861]. The most important [61] of these local parameters is the local volume fraction $\phi_{l}$ which describes by how much free volume an individual particle is surrounded. $\phi_{l}$ is computed by dividing the volume of the particle by the

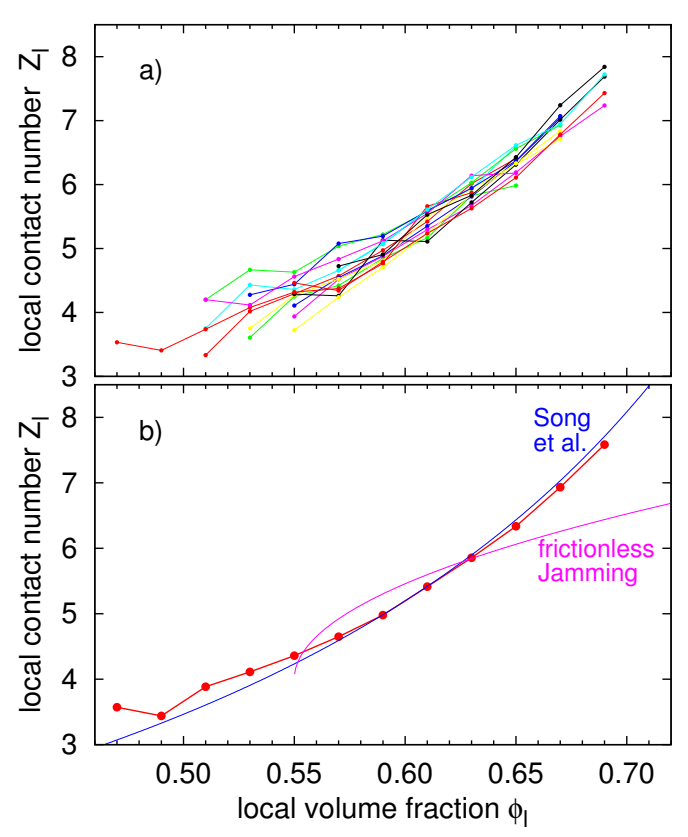

Figure 6. Understanding contact numbers in sphere packings requires a local approach. a) The average local contact number $Z_{l}$ of individual spheres, measured by X-ray tomography and averaged in local volume fraction $\phi_{l}$ bins of size 0.02 . Data corresponds to 15 different sphere packings with global volume fractions $\phi_{g}$ in the range from 0.56 to 0.625 . Within experimental scatter, $Z_{l}$ depends only on $\phi_{l}$, not on $\phi_{g}$. b) The red dots correspond to a bin-wise average of all data shown in panel a. The local mean field theory by Song et al. (eq. 3, no fit parameter) provides a fair description of the data. This can not be said about the local interpretation of the scaling law for frictionless, compressed spheres (eq. 2, one fit parameter). From [61].

volume of its Voronoi cell (a tessellation method assigning all points in space to the closest particle). However, for a complete local description more parameters such as the shape of the Voronoi cells [62] or the fabric anisotropy [63] are needed.

Figure 6 substantiates this claim for the necessity of local theories. Panel a shows that the number of contacts $Z_{l}$ an individual particle will form does only depend on its own $\phi_{l}$, not the $\phi_{g}$ value of the packing it resides in. Figure 6 b) demonstrates that $Z_{l}$ can be well explained by the local theory presented in [31], which predicts:

$$
Z=\frac{2 \sqrt{3} \phi_{l}}{1-\phi_{l}}
$$

A local reinterpretation of equation 2 for frictional systems $\left(\phi_{g}\right.$ becomes $\phi_{l}, Z_{i s o}^{0}$ becomes $Z_{i s o}^{\mu}, \phi_{i s o}$ becomes $\left.\phi_{R L P}\right)$ fails to describe the experimental data.

\section{Friction is one reason for history dependence in granular systems.}

A number of experiments demonstrate history dependent behavior in granular materials: Two seemingly identical packings, which only differ in their preparation histories, 

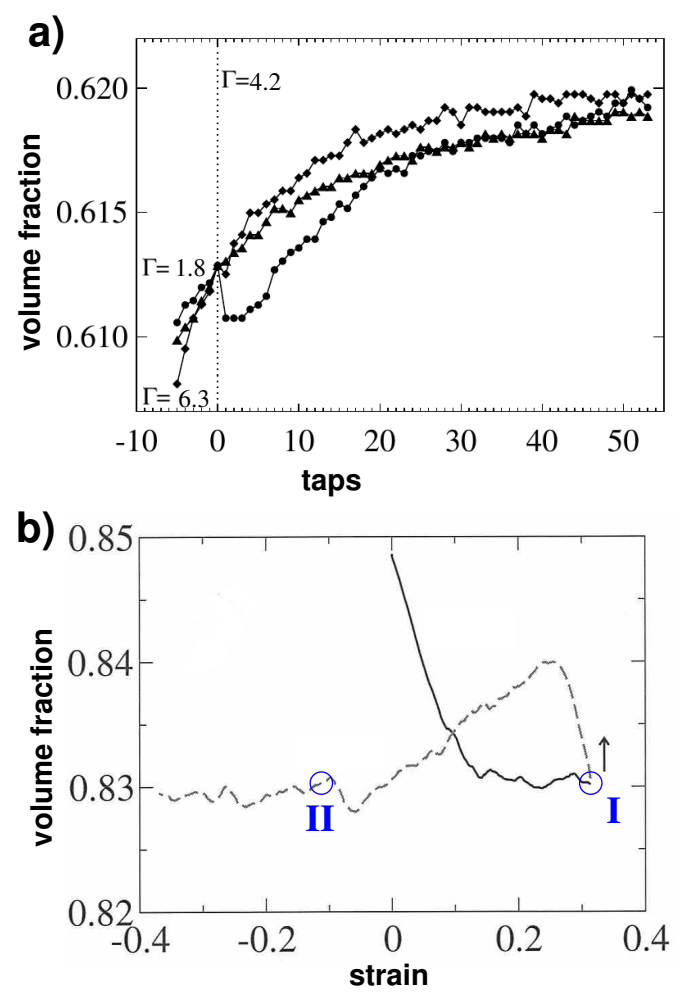

Figure 7. History dependence in granular systems. a) Three samples of glass beads are compactified to the same value of $\phi_{g}=0.613$ using three different initial tapping strengths. At this point (vertical dotted line) the tapping strength is set to the same value of $4.2 \mathrm{~g}$ in all three experiments. The system does however respond differently depending on its preparation history. From [64]. b) Volume fraction alone is not sufficient to characterize dilatancy onset. In this contact dynamics simulations an initially dense system of discs has been sheared long enough to dilate to its critical state (solid line). When then the shear direction is reversed (dashed line), the system responds first by compaction before it dilates again. This implies that the packings at points I and II have identical volume fractions but respond differently to shear in the same direction. From [65].

respond either differently to an external excitation, or they differ in some of their not immediately obvious mechanical properties.

An example of the first type is shown in figure 7a: three samples are compactified to the same volume fraction $\phi_{0}$ but using three different driving strengths $\Gamma_{i}$. When these samples at $\phi_{0}$ are then driven with the same strength $\Gamma_{0}$, their response depends on the past $\Gamma_{i}$, not $\Gamma_{0}$ [64]. Similar results can be obtained for periodically shearing glass spheres in a parallelepiped shear cell [66] or going to large strains in a simple shear cell (fig. 7b) [65].

Examples of the second type of history dependence include how the pressure distribution below a sandpile depends on its preparation history: If the sand rained down from a large sieve, the maximum pressure at the bottom plate will be below the tip; which is the point with the largest column of sand on top. However, if the sand flowed out of a small funnel opening, which means that the pile grew from many downhill avalanches, the maximum pres- sure at the bottom plate will be at a ring with a diameter of roughly one third of the total pile diameter [67]. An similar example is the history dependence that exists in the so called Janssen effect: The pressure at the bottom of a cylindrical column filled with grains will be lower than the total weight of the grains because tangential forces at the sidewalls carry a part of the load. The amount of this reduction will again depend on the preparation history [68]. Finally it has also been shown numerically, that the number of contacts formed in a packing depends on the preparation history [69].

History dependence does also exist in frictionless packings (see $[28,51]$ for a novel approach how the jamming volume fraction can be used as a state variable to characterize the history). However, most of the examples listed above seem to require friction. Either because the extra degrees of freedom allow variability in the contact number or the geometric fabric formed by the contacts (pressure distribution at the sand pile bottom, shear response at critical state). Or because the memory of a previous state can be encoded as a particular configuration in the force phase space spanned by hyperstaticity (Janssen).

In all examples discussed here the apparent identity of the initial states has only been established in terms of global variables such as shape of the sample and $\phi_{g}$. In fact, in all these cases history dependence can also be viewed as another name for: "we do not know all relevant parameters which characterize the system".

\section{Granular matter is rarely spherical.}

Most of the readers will have heard some variant of the "spherical cow in vacuum" joke [72]. But in fact there are not only good reasons for theorists to use spheres as a first approximation, also numerical scientists appreciate the easy collision detection algorithm coming with spheres. And experimentalist like spheres because they are the only monoschematic particles (all particles have the same shape) which are easily available in large quantities. Being monoschematic is a big advantage during image processing where the a priori knowledge of their shape helps to identify the individual particles [73].

Still, real world granular materials are basically always non-spherical in shape. This adds additional complexity which every theory suitable for practical purposes will need to take into account. Figure 8 discusses some of this complexity using packings of tetrahedra as an example. Contrary to spheres, tetrahedra can form four different types of contacts, cf. figure $8 \mathrm{~b}$. This has important consequences for the pair correlation function shown in figure $8 \mathrm{a}$. Because perfectly aligned face-to-face contacts are less frequent than slightly shifted face-to-face or low angle face-to-edge contacts, the closest possible distance between two particles is not the most likely contact configuration [70]. The different shape of the first peak of $g(r)$ brings as a consequence that scaling laws developed for compressible sphere packings [7, 74], such as eq. 2, will not work for compressible tetrahedra. Moreover, figure $8 \mathrm{c}$ ) shows, that tetrahedra packings are even stronger hyperstatic and history-dependent than sphere packings. 
a)
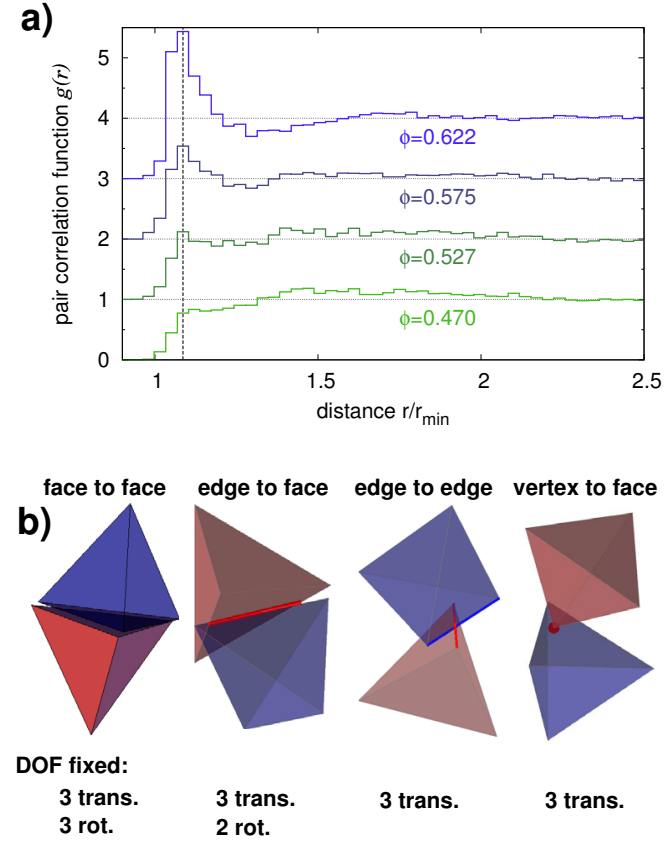

c)

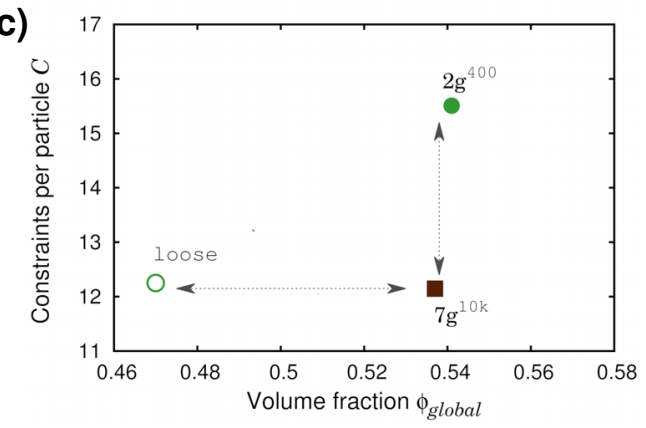

Figure 8. Packings of tetrahedra differ from sphere packings. a) The pair correlation functions of experimental tetrahedra packings shows that contrary to spheres the shortest possible distance $r_{\text {min }}$ (approximately 0.408 times the side-length) is not the most likely distance between individual particles (indicated by a vertical dashed line) [70]. Offsets have been added for improved readability. b) Tetrahedra form four different types of contacts, which block different numbers of translational and rotational degrees of freedom. For the purpose of defining a distance to isostaticity we need to determine the total number of constraints per particle $C$ blocked by the combination of all four contact types. c) Isostaticity corresponds to $C=6$ (each tetrahedra has three rotational and three translational DOF). Even the loosest tetrahedra packings have twice the constraint number needed for isostaticity, which means that tetrahedra packings are much more hyperstatic than sphere packings. Moreover do tetrahedra packings exhibit a strong history dependence. Using different tapping protocols it is possible to create pairs of packing which differ strongly in only one of the two variables $C$ and $\phi_{g}$ [71].

Experimentally, one way of moving away from the spherical cow paradigm is to take advantage of the quickly improving 3D printing technology to create large samples of monoschematic but non-spherical particles [75, 76]. Alternatively, we can directly use natural materials such as sand and improve our 3D image processing to obtain trust- worthy segmentation results [77]. Additionally, there is also progress towards theories for non-spherical particles $[59,60]$.

\section{Conclusion}

Frictionless spheres are a great model for emulsions, foams, glasses, and colloids. They give reasonable results for granular gases and describe even glassy behavior in driven granular systems. But a frictionless granular packing is a a self-contradicting statement, describing a theoretical model that for the most part has outlived its usefulness.

\section{Acknowledgments}

The ideas in this article have taken shape in many invaluable discussions with colleagues: Vasili Baranau, Karen Daniels, Max Neudecker, Charles Radin, Fabian Schaller, Gerd Schröder-Turk, Harry Swinney, Brian Tighe, SongChuan Zhao.

\section{References}

[1] M. Schröter, D.I. Goldman, H.L. Swinney, Phys. Rev. E 71, 030301 (2005)

[2] G.R. Farrell, K.M. Martini, N. Menon, Soft Matter 6, 2925 (2010)

[3] F.P. Bowden, D. Tabor, Friction (Anchor Books, Garden City, 1973)

[4] J.A. Dijksman, H. Zheng, R.P. Behringer, in Powders $\mathcal{E}$ Grains (2013), Vol. 1542 of AIP Conf. Proc., pp. 457-460

[5] J. Dijksman, private communication (2017)

[6] G.J. Gao, J. Blawzdziewicz, C.S. O’Hern, M. Shattuck, Phys. Rev. E 80, 061304 (2009)

[7] M. van Hecke, J. Phys.: Condens. Matter 22, 033101 (2010)

[8] B.P. Tighe, J.H. Snoeijer, T.J.H. Vlugt, M.v. Hecke, Soft Matter 6, 2908 (2010)

[9] K. Shundyak, M. van Hecke, W. van Saarloos, Phys. Rev. E 75, 010301 (2007)

[10] L.E. Silbert, D. Ertaş, G.S. Grest, T.C. Halsey, D. Levine, Phys. Rev. E 65, 031304 (2002)

[11] H.P. Zhang, H.A. Makse, Phys. Rev. E 72, 011301 (2005)

[12] S. Henkes, M. van Hecke, W. van Saarloos, Europhys. Lett. 90, 14003 (2010)

[13] S.F. Edwards, R.B.S. Oakeshott, Physica A 157, 1080 (1989)

[14] A. Mehta, S.F. Edwards, Physica A 157, 1091 (1989)

[15] A.V. Anikeenko, N.N. Medvedev, Phys. Rev. Lett. 98, 235504 (2007)

[16] C. Radin, J. Stat. Phys. 131, 567 (2008)

[17] Y. Jin, H.A. Makse, Physica A 389, 5362 (2010)

[18] S.C. Kapfer, W. Mickel, K. Mecke, G.E. SchröderTurk, Phys. Rev. E 85, 030301 (2012) 
[19] N. Francois, M. Saadatfar, R. Cruikshank, A. Sheppard, Phys. Rev. Lett. 111, 148001 (2013)

[20] V. Baranau, U. Tallarek, Soft Matter 10, 3826 (2014)

[21] V. Baranau, U. Tallarek, Soft Matter 10, 7838 (2014)

[22] S. Torquato, F.H. Stillinger, J. App. Phys. 102, 093511 (2007)

[23] G. Parisi, F. Zamponi, Rev. Mod. Phys. 82, 789 (2010)

[24] D. Asenjo, F. Paillusson, D. Frenkel, Phys. Rev. Lett. 112, 098002 (2014)

[25] S. Martiniani, K.J. Schrenk, J.D. Stevenson, D.J. Wales, D. Frenkel, Phys. Rev. E 93, 012906 (2016)

[26] C.S. O’Hern, L.E. Silbert, A.J. Liu, S.R. Nagel, Phys. Rev. E 68, 011306 (2003)

[27] M. Pica Ciamarra, M. Nicodemi, A. Coniglio, Soft Matter 6, 2871 (2010)

[28] N. Kumar, S. Luding, Granul. Matter 18, 58 (2016)

[29] G.Y. Onoda, E.G. Liniger, Phys. Rev. Lett. 64, 2727 (1990)

[30] M. Jerkins, M. Schröter, H.L. Swinney, T.J. Senden, M. Saadatfar, T. Aste, Phys. Rev. Lett. 101, 018301 (2008)

[31] C. Song, P. Wang, H.A. Makse, Nature 453, 629 (2008)

[32] L.E. Silbert, Soft Matter 6, 2918 (2010)

[33] G.W. Delaney, J.E. Hilton, P.W. Cleary, Phys. Rev. E 83, 051305 (2011)

[34] Y. Srebro, D. Levine, Phys. Rev. E 68, 061301 (2003)

[35] V. Baranau, S.C. Zhao, M. Scheel, U. Tallarek, M. Schröter, Soft Matter 12, 3991 (2016)

[36] C. Briscoe, C. Song, P. Wang, H.A. Makse, Phys. Rev. Lett. 101, 188001 (2008)

[37] S. McNamara, P. Richard, S.K. de Richter, G. Le Caer, R. Delannay, Phys. Rev. E 80, 031301 (2009)

[38] S.C. Zhao, M. Schröter, Soft Matter 10, 4208 (2014)

[39] E.R. Nowak, J.B. Knight, E. Ben-Naim, H.M. Jaeger, S.R. Nagel, Phys. Rev. E 57, 1971 (1998)

[40] P. Ribière, P. Richard, P. Philippe, D. Bideau, R. Delannay, Eur. Phys. J. E 22, 249 (2007)

[41] B. Andreotti, Y. Forterre, O. Pouliquen, Granular Media (Cambridge University Press, 2013)

[42] M. Schröter, S. Nägle, C. Radin, H.L. Swinney, Europhys. Lett. 78, 44004 (2007)

[43] A.J. Kabla, T.J. Senden, Phys. Rev. Lett. 102, 228301 (2009)

[44] N. Gravish, P.B. Umbanhowar, D.I. Goldman, Phys. Rev. Lett. 105, 128301 (2010)

[45] P. Umbanhowar, D.I. Goldman, Phys. Rev. E 82, 010301 (2010)

[46] J. Métayer, D.J. Suntrup III, C. Radin, H.L. Swinney, M. Schröter, Europhys. Lett. 93, 64003 (2011)

[47] N. Mueggenburg, Phys. Rev. E 85, 041305 (2012)

[48] D. Bi, J. Zhang, B. Chakraborty, R.P. Behringer, Nature 480, 355 (2011)

[49] J. Ren, J.A. Dijksman, R.P. Behringer, Phys. Rev. Lett. 110, 018302 (2013)
[50] H.A. Vinutha, S. Sastry, Nature Phys. 12, 578 (2016)

[51] S. Luding, Nature Phys. 12, 531 (2016)

[52] G. Katgert, M. van Hecke, Europhys. Lett. 92, 34002 (2010)

[53] S. Utermann, P. Aurin, M. Benderoth, C. Fischer, M. Schröter, Phys. Rev. E 84, 031306 (2011)

[54] T.S. Majmudar, M. Sperl, S. Luding, R.P. Behringer, Phys. Rev. Lett. 98, 058001 (2007)

[55] C. Coulais, R.P. Behringer, O. Dauchot, Soft Matter 10, 1519 (2014)

[56] F. Lechenault, F.d. Cruz, O. Dauchot, E. Bertin, J. Stat. Mech. 2006, P07009 (2006)

[57] S.C. Zhao, S. Sidle, H.L. Swinney, M. Schröter, Europhys. Lett. 97, 34004 (2012)

[58] T. Aste, M. Saadatfar, T.J. Senden, J. Stat. Mech.: Theory and Exp. 2006, P07010 (2006)

[59] A. Baule, R. Mari, L. Bo, L. Portal, H.A. Makse, Nat. Commun. 4, 2194 (2013)

[60] A. Baule, H.A. Makse, Soft Matter 10, 4423 (2014)

[61] F.M. Schaller, M. Neudecker, M. Saadatfar, G.W. Delaney, G.E. Schröder-Turk, M. Schröter, Phys. Rev. Lett. 114, 158001 (2015)

[62] G.E. Schröder-Turk, W. Mickel, M. Schröter, G.W. Delaney, M. Saadatfar, T.J. Senden, K. Mecke, T. Aste, Europhys. Lett. 90, 34001 (2010)

[63] F. Radjai, in Powders E Grains (2009), Vol. 1145 of AIP Conf. Proc., pp. 35-42

[64] C. Josserand, A.V. Tkachenko, D.M. Mueth, H.M. Jaeger, Phys. Rev. Lett. 85, 3632 (2000)

[65] F. Radjaï, S. Roux, in The Physics of Granular Media, edited by H. Hinrichsen, D.E. Wolf (WileyVCH, Weinheim, 2004), pp. 165-187

[66] M. Nicolas, P. Duru, O. Pouliquen, Eur. Phys. J. E 3, 309 (2000)

[67] L. Vanel, D. Howell, D. Clark, R.P. Behringer, E. Clément, Phys. Rev. E 60, R5040 (1999)

[68] L. Vanel, E. Clément, Eur. Phys. J. B 11, 525 (1999)

[69] I. Agnolin, J.N. Roux, Phys. Rev. E 76, 061302 (2007)

[70] M. Neudecker, S. Ulrich, S. Herminghaus, M. Schröter, Phys. Rev. Lett. 111, 028001 (2013)

[71] N.N. Thyagu, F. Schaller, S. Weis, M. Neudecker, M. Schröter, arXiv:1501.04472 (2015)

[72] https://www.youtube.com/watch? $\mathrm{v}=\mathrm{oUwlEdz} 42 \times 0$ (2013)

[73] S. Weis, M. Schröter, submitted to Rev. Sci. Instr. (2017), http://arxiv.org/abs/arXiv:1612.06639

[74] A.J. Liu, S.R. Nagel, Ann. Rev. Cond. Matt. Phys. 1, 347 (2010)

[75] A.G. Athanassiadis, M.Z. Miskin, P. Kaplan, N. Rodenberg, S.H. Lee, J. Merritt, E. Brown, J. Amend, H. Lipson, H.M. Jaeger, Soft Matter 10, 48 (2013)

[76] C. Scholz, S. D’Silva, T. Pöschel, New J. Phys. 18, 123001 (2016)

[77] I. Vlahinić, E. Andò, G. Viggiani, J.E. Andrade, Granul. Matter 16, 9 (2014) 